Supporting information

\title{
Bioinspired Polyelectrolyte-Assembled \\ Graphene-Oxide-Coated C18 Composite Solid-Phase \\ Microextraction Fibers for in Vivo Monitoring of Acidic \\ Pharmaceuticals in Fish
}

Junlang Qiu, Guosheng Chen, Shuqin Liu, Tianlang Zhang, Jiayi Wu, Fuxin Wang,

Jianqiao Xu, Yan Liu, Fang Zhu*, and Gangfeng Ouyang*

MOE Key Laboratory of Aquatic Product Safety/KLGHEI of Environment and Energy

Chemistry, School of Chemistry and Chemical Engineering, Sun Yat-sen University,

Guangzhou 510275, China

${ }^{*}$ Corresponding author. Tel. \& Fax: +86-20-84110845

E-mail: ceszhuf@mail.sysu.edu.cn (F. Zhu); cesoygf@mail.sysu.edu.cn (G. Ouyang).

Page: 8

Text: 2

Figure: 3

Table: 2 
Method S1. LC-MS/MS analysis: high performance liquid chromatography-tandem mass spectrometry (MS/MS) analysis was based on an Agilent 1260 HPLC system (Agilent Technologies, CA, USA) coupled to an AB Sciex Triple Quad 4500 triple-quadrupole tandem mass spectrometer with an ESI source in both positive and negative ion mode (Applied Biosystems/MDS Sciex, MA, USA). A Zorbax SB-C18 column $(2.1 \mathrm{~mm} \times 150 \mathrm{~mm}, 3.6 \mu \mathrm{m}$, Agilent Technologies, CA, USA) was used for separation. Aqueous solution containing $0.01 \%$ formic acid (A) and acetonitrile (B) were used as mobile phases, the flow rate was set at $500 \mu \mathrm{L} \cdot \mathrm{min}^{-1}$. The initial gradient of $90 \%$ A ramped to $2.5 \% \mathrm{~A}$ in 5 min and kept for 4 min, then ramped back to $90 \% \mathrm{~A}$ in $0.1 \mathrm{~min}$ and kept for $4.9 \mathrm{~min}$ to balance the column pressure. Transitions were monitored as follows: positive ion mode: CAR $m / z$ 237.1/194.1, FLU $m / z$ 310.1/148.0, FLU-d5 m/z 315.2/153.1; negative ion mode: MEF $m / z$ 239.9/195.8, FLUF $m / z$ 279.9/235.8, FLUR 243.1/198.7, KET 253.1/208.6, TOL 260.0/215.6, GEM 249.0/120.5, NAP 229.0/184.5, IBU 205.0/160.7. 
Method S2. A fish was killed and approximately $100 \mathrm{mg}$ of dorsal-epaxial muscle was collected in a centrifugation tube. Aliquots of $100 \mu \mathrm{L}$ of ascorbic acid solution $(0.1 \mathrm{M})$ and $400 \mu \mathrm{L}$ of ice-cold methanol were added in the centrifugation tube; then, the tube was sonicated in an ice bath for $20 \mathrm{~min}$ and centrifuged at $3500 \mathrm{rpm}$ for 30 min. The supernatant was transferred to an amber vial and dried under $60{ }^{\circ} \mathrm{C}$ in a nitrogen stream. The residue was dissolved in an aliquot of $200 \mu \mathrm{L}$ of methanol, and $100 \mu \mathrm{L}$ of the solution was transferred to the inset tube in an amber vial for LC-MS/MS analysis. 
Figure S1. Deployment of the novel SPME fiber in fish muscle. Deploy the custom-made fiber under the guidance of steel needle (A), remove the steel needle and expose the custom-made fiber in the biological matrix (B), carefully put back the steel needle to the matrix at the end of sampling (C), and remove the custom-made fiber (D).

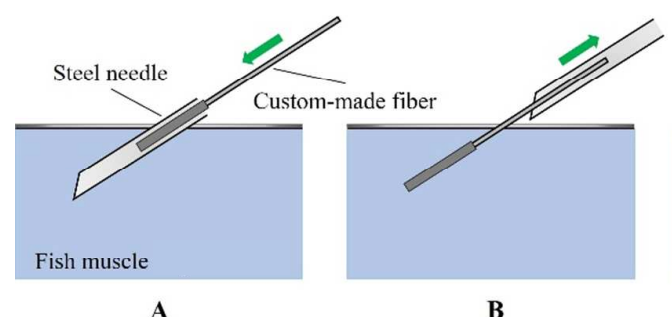

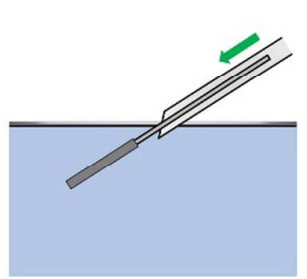

C

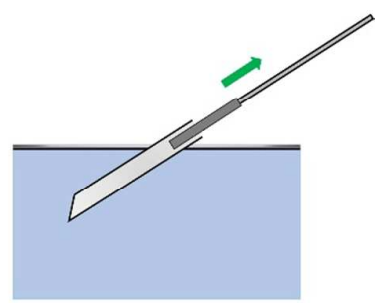

D 
Figure S2. The image of modified (left) and unmodified (right) fibers.

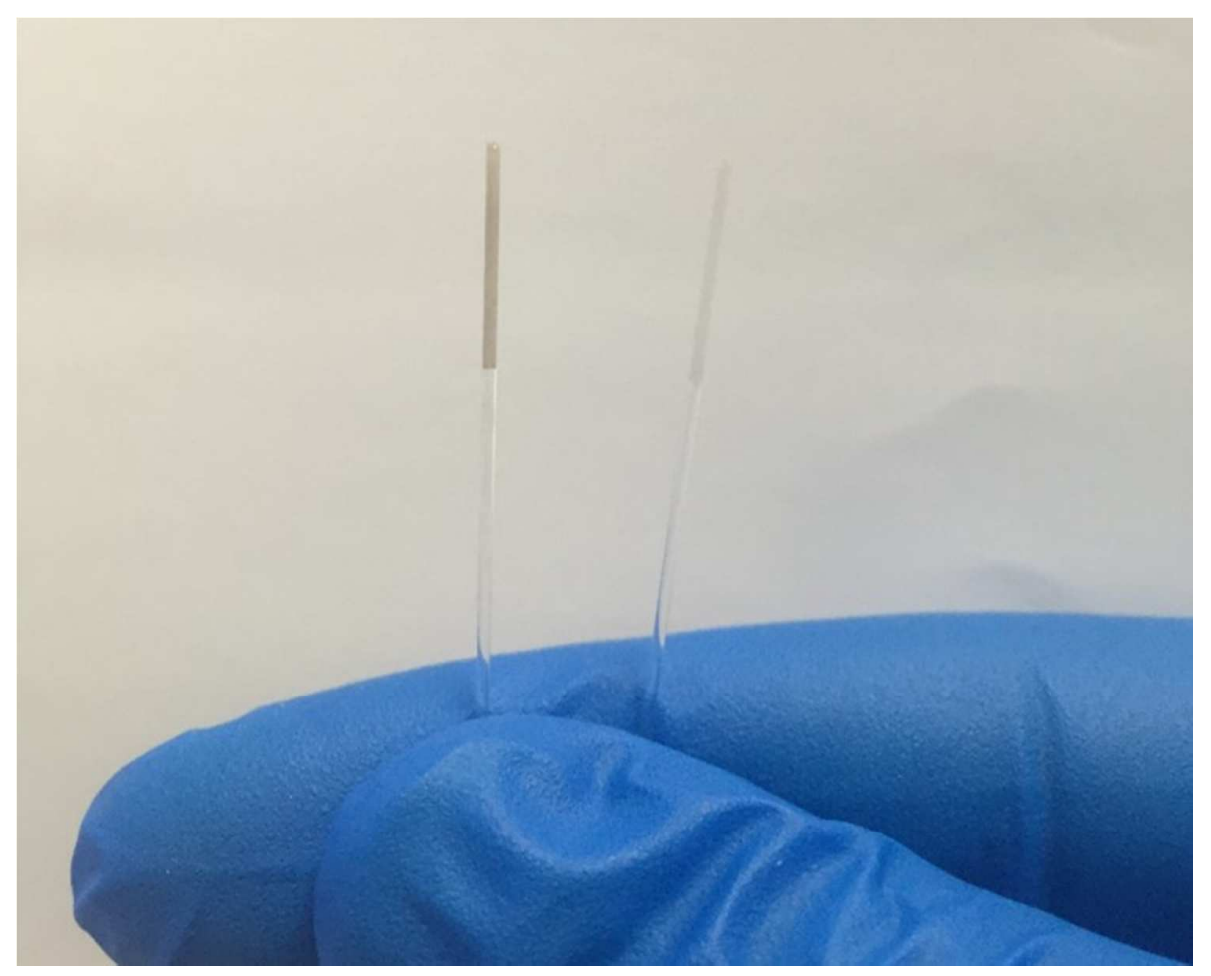


Figure. S3. MALDI-TOF MS analysis of the desorption solvent of the modified (A) and unmodified (B) fibers that exposed in blank fish dorsal-epaxial muscle for ten minutes. DHB and SA were used as the matrices for the $\mathrm{m} / \mathrm{z}$ ranges of $300-5000 \mathrm{Da}$ and 5000-80000 Da, respectively.
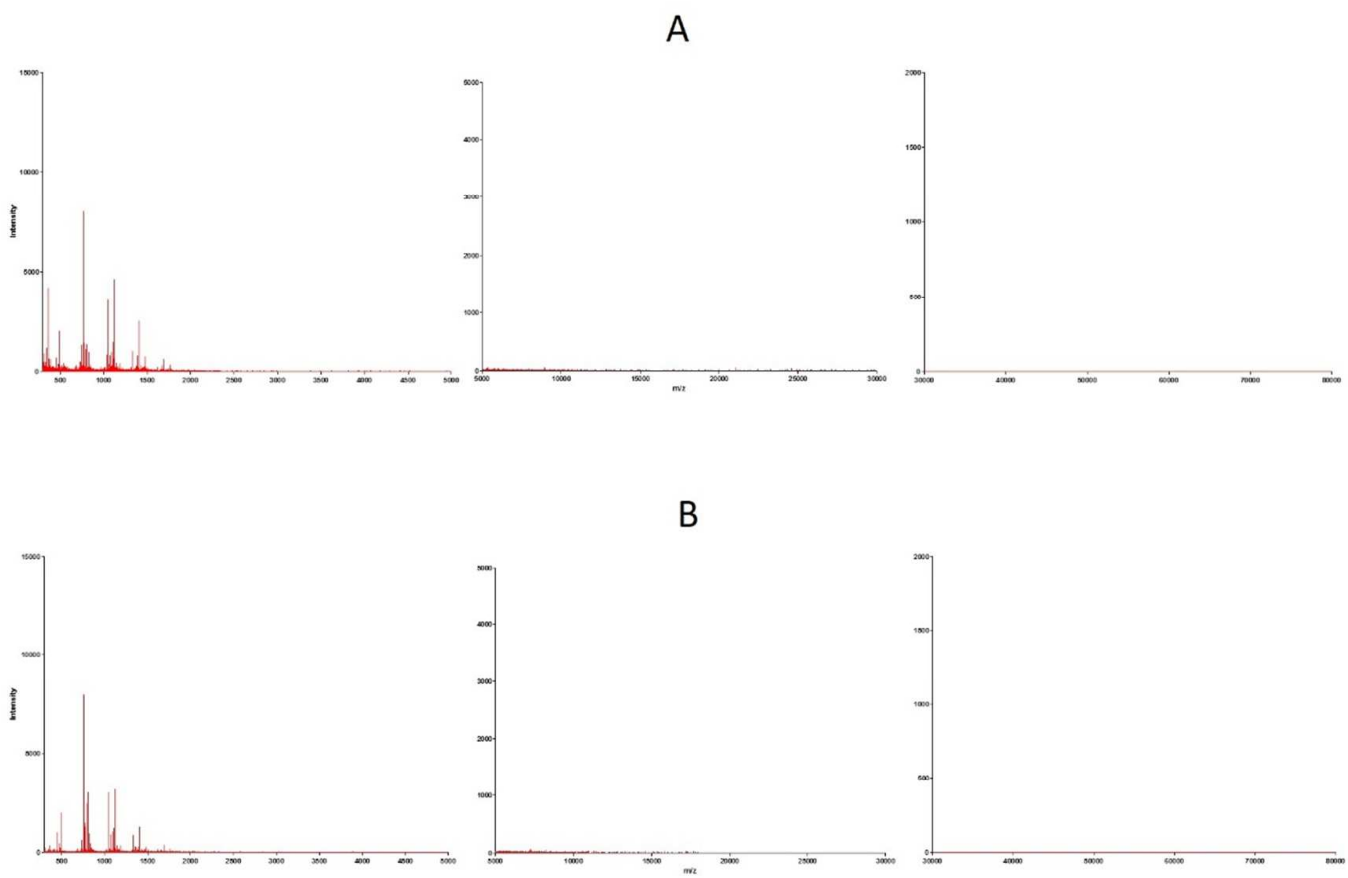
Table S1. Regression slopes $(k)$ of curves (signal to concentration, the units were omitted) derived from the matrix-free and matrix-impacted (fish) solutions of modified $(m)$ and unmodified $(u)$ fibers. The concentration ranges were both from 1 to $100 \mathrm{ng} \cdot \mathrm{mL}^{-1} \cdot R^{2}$ was the linear regression coefficient.

\begin{tabular}{|c|c|c|c|c|c|c|c|c|}
\hline \multirow{3}{*}{ Analytes } & \multicolumn{4}{|c|}{ Modified $(m)$} & \multicolumn{4}{|c|}{ Unmodified $(u)$} \\
\hline & \multicolumn{2}{|c|}{ Matrix-free } & \multicolumn{2}{|c|}{ Matrix-impacted } & \multicolumn{2}{|c|}{ Matrix-free } & \multicolumn{2}{|c|}{ Matrix-impacted } \\
\hline & $k_{i-m}$ & $R^{2}$ & $k_{f-m}$ & $R^{2}$ & $k_{i-u}$ & $R^{2}$ & $k_{f-u}$ & $R^{2}$ \\
\hline CAR & 847033 & 0.9973 & 885007 & 0.9936 & 856369 & 0.9944 & 875232 & 0.9928 \\
\hline FLU & 62639 & 0.9963 & 62184 & 0.9938 & 64362 & 0.9978 & 63265 & 0.9941 \\
\hline MEF & 598652 & 0.9977 & 618260 & 0.9973 & 585623 & 0.9981 & 602369 & 0.9966 \\
\hline FLUF & 956307 & 0.9989 & 886165 & 0.9955 & 923265 & 0.9969 & 896359 & 0.9935 \\
\hline FLUR & 69920 & 0.9989 & 74487 & 0.9982 & 72668 & 0.9973 & 70236 & 0.9971 \\
\hline KET & 175562 & 0.9985 & 180575 & 0.9984 & 196365 & 0.9981 & 185623 & 0.9962 \\
\hline TOL & 948242 & 0.9999 & 911148 & 0.9934 & 929741 & 0.9996 & 936288 & 0.9929 \\
\hline GEM & 912021 & 0.9941 & 929248 & 0.9953 & 902583 & 0.9979 & 896357 & 0.9968 \\
\hline NAP & 109411 & 0.9926 & 108852 & 0.9972 & 111236 & 0.9983 & 120369 & 0.9948 \\
\hline IBU & 233154 & 0.9996 & 229975 & 0.9929 & 212569 & 0.9968 & 202369 & 0.9951 \\
\hline
\end{tabular}


Table S2. Sampling rates of the tested pharmaceuticals determined in fish muscle.

\begin{tabular}{ccc}
\hline Analytes & Mean $/(\mu \mathrm{g} / \mathrm{min})$ & $\mathrm{RSD} / \%$ \\
\hline FLU & 1349.58 & 18.17 \\
MEF & 487.60 & 29.96 \\
FLUF & 746.39 & 14.96 \\
FLUR & 1189.83 & 25.94 \\
KET & 734.69 & 23.52 \\
TOL & 1150.35 & 22.73 \\
GEM & 666.43 & 14.25 \\
NAP & 605.67 & 14.09 \\
IBU & 835.35 & 17.69 \\
\hline
\end{tabular}

